\title{
On the Connectivity of Hierarchical Directional Optical Sensor Networks
}

\author{
Unoma Ndili Okorafor and Deepa Kundur \\ Department of Electrical \& Computer Engineering \\ Texas A \& M University, College Station, Texas 77843-3124 \\ Email: \{unondili, deepa\}@ece.tamu.edu
}

\begin{abstract}
In this paper, we investigate the fundamental property of connectivity in a hierarchical ad-hoc optical sensor network (OSN) communicating with broad beam lasers. The OSN is modeled as a random sector graph, in which the randomly deployed sensors can send data within a contiguous and randomly oriented sector of communication. Hierarchy is achieved by employing passive and active free space optical devices. Connectivity analysis in this hierarchical network model is vital in order to assess the feasibility of routing, security and network protocols. To this end, this paper provides probabilistic bounds on network parameters for hierarchical connectivity in OSNs, using a novel approach that directly considers directionality. In particular, we prove probabilistic bounds on the jointly minimum radius of communication as well as angle of the communication sector (i.e., beam width) required for network connectivity.
\end{abstract}

\section{INTRODUCTION}

Optical sensor networks (OSNs) represent a class of sensor networks that communicate using free space optics (FSO) with a directed and randomly oriented sector of communication. OSNs are rapidly gaining visibility due to the advantages that FSO offers over traditional omni-directional and directional RF based techniques [1]-[4]. The flat network of the OSN has been modeled as a directed random sector graph [1], and hierarchical clustering is easily achieved by utilizing both passive and active FSO devices [2]-[4].

In an ad-hoc sensor network, connectivity is of particular significance in order to maintain communication among nodes. This is especially true for OSNs in which directional communication has distinct characteristics and effects on connectivity, coverage, security, quality of service and routing that warrant novel analysis and insights. To this end, this paper provides to the best of our knowledge, the first known analysis of network connectivity for the flat and hierarchical directional OSN setup. Our analysis is a first step to studying the feasibility of the OSN, and the network layer protocols that operate on them. In contrast to several known connectivity analysis [5][7], our contribution leverages directionality.

For our work, connectivity for the directional OSN is defined in the conventional sense: there exists a directed path from any node to every other node in the network. Let us now define the OSN as forward-connected if every node in the network has a non-empty forward neighborhood, so that each node can send data to another node, (see Figure 1(a)). Similarly, the OSN is backward-connected if every node is covered by the communication sector of at least one other node, so that every node can receive data from some other node, i.e., no node is backward-isolated, (see Figure 1(b)). We note that directed graphs may be forward-connected, but not backward-connected, and vice versa, and a necessary (albeit insufficient) condition for the connectivity of the OSN is that the network is both forward and backward connected, defined as fb-connected. An fb-connected network may not be connected due to possible partitioned components, and lack of bidirectional paths between all node pairs (e.g. in the fbconnected graph of Figure 1(c) the path $S_{i}$ to $S_{j}$ (denoted $S_{i} \rightarrow S_{j}$ ) exists, however path $S_{j} \rightarrow S_{i}$ does not. We define a $B S$-circuit as a directed path which starts and ends at the base station through a sequence of nodes. Hierarchical connectivity implies that all nodes have both a send and receive path to/from the base station, and underscores the main objective of a sensor network.

In this paper, we show that the necessary and sufficient conditions for OSN connectivity is that the OSN graph is fb-connected, and every node is contained in at least one BS-circuit. We derive probabilistic bounds on forward- and backward-connectivity in terms of node density, angle $\alpha$ and radius $r$ of a nodes' communication sector. Results of our paper gives expressions for the jointly minimal values for $\alpha$ and $r$ which enable us derive further bounds on network parameters for flat and hierarchical connectivity. Our paper is organized as follows: Section 2 discusses related work, while Section 3 presents preliminaries and network setup. In Section 4 we present our main results for connectivity in the flat OSN. Section 5 discusses hierarchical connectivity, while Section 6 presents concluding remarks.

\section{RELATED WORK}

Several connectivity analysis have been proposed based on the random geometric graph (RGG) model as the basic network model for packet radio sensor networks [6]-[12]. The RGG is an $r$-radius model in which all nodes employ the same radio power, so that each node $S_{i}$ can establish a direct link with any other node within a ball $B\left(S_{i}, r(n)\right)$ with center $S_{i}$ and fixed radius $r(n)$, where $n$ is the number of nodes in the network. Gupta \& Kumar [6] show that for such a network uniformly deployed in a disc of unit area in $\mathcal{R}^{2}$, if $r(n)=$ $\sqrt{(\log (n)+c(n)) / \pi n}$ ), the network is a.a.s. connected (as $n \rightarrow \infty$ with probability one), if and only if $\lim _{n \rightarrow \infty} c(n)=$ $+\infty$, where $c(n)$ is a constant. The probabilistic approach 


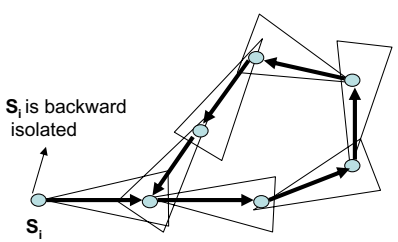

Forward-connected OSN

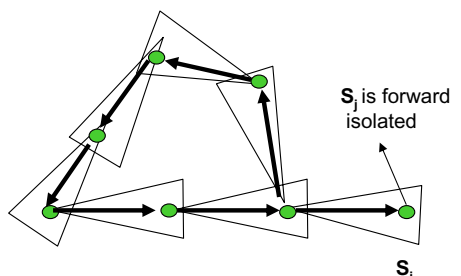

Backward-connected OSN

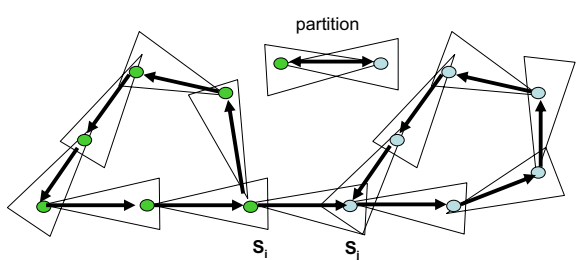

fb-connected OSN

Fig. 1. (a) A forward-connected OSN which is not backward-connected since node $S_{i}$ cannot receive data from any other node in the network. (b) A backward-connected OSN which is not forward-connected. Node $S_{j}$ cannot send data to any other node in the network. (c) An fb-connected OSN which is still not connected since $S_{j}$ cannot send data to $S_{i}$, and a partition exists in the network.

of [7] employs nearest neighbor methods to show that for a network with node density $\rho$, with probability at least $p$, no node is isolated if $r(n) \geq \sqrt{-\ln \left(1-p^{1 / n}\right) / \rho \pi}$.

Hierarchical connectivity analysis for sensor networks has received almost no attention in the literature. We assume this is so because of the underlying assumption that if the flat network of the RGG is connected, so should the hierarchical network. In addition, the major motivation for hierarchy in the past has been clustering for scalability, and not to improve connectivity. In OSNs, due to directionality, flat network connectivity is more difficult to achieve. If we assume a virtual connection grid exists between all clusterhead nodes (via the base station), then hierarchy may be employed to improve connectivity. In this case, factors such as the density and distribution of the cluster heads affect connectivity.

Diaz, Petit \& Serna [1] provide connectivity analysis for directional OSNs based on asymptotic reasoning leveraging the work in [6]. They show that if the underlying RGG is asymptotically almost surely (a.a.s.) connected with high probability (w.h.p.), then w.h.p., any link in the RGG can be emulated with at most four links in the OSN. Our work differs significantly from [1] as we apply probabilistic reasoning. Our results are useful in setting practical values for network parameters such as $r, \alpha$ and $n$.

\section{Preliminaries: Network Model}

\section{A. Flat Network Setup}

Consider an OSN in which a set $\left\{\mathcal{S}_{n}\right\}$ of $n$ nodes, indexed as $S_{i}: i=1,2, \cdots n$, are randomly and densely deployed in a given area $[0,1]^{2}$ according to a uniform distribution $\sim U[0,1]^{2}$. Such a random deployment may occur in a scenario where priori knowledge of the field is not available, or may be the result of the deployment strategies. Let $I($. be an information assignment function on $\left\{S_{n}\right\}$, where $I$ is a positive real valued mapping from $\left\{S_{n}\right\}$ to the 3-tuple as:

$$
I\left(S_{n}\right):\left\{S_{n}\right\} \rightarrow(\mathbf{x}, \mathbf{y}, \Theta)
$$

$\mathbf{x}=\left(x_{1}, x_{2}, \cdots x_{n}\right)$ and $\mathbf{y}=\left(y_{1}, y_{2}, \cdots y_{n}\right)$ represent the $x-y$ position coordinates of $\left\{S_{n}\right\}$, such that $x_{i}, y_{i} \sim U(0,1] \forall i$. We denote $\operatorname{Pos}_{i}=\left(x_{i}, y_{i}\right)$ as the position of node $S_{i}$. Under this assumption, the number of nodes in a given area can be modeled by a stationary 2-dimensional Poisson point process Poisson $(n)$, with density equal to the number of sensors per unit area [8], [13], [14]. The orientation vector $\Theta=$
$\left(\Theta_{1}, \Theta_{2}, \cdots \Theta_{n}\right)$ of $\left\{S_{n}\right\}$ is modeled as $\Theta_{i} \sim U(0,2 \pi] \forall i$. $I\left(S_{n}\right)$ is called the Information on $\left\{S_{n}\right\}$.

Each node $S_{i}$ can orient its transmitting optical laser within a contiguous angular scanning region $\frac{-\alpha}{2}+\Theta_{i} \leq \Phi_{i} \leq$ $\frac{+\alpha}{2}+\Theta_{i}$. Following the model in [1], [2] and as depicted in Figure 2(a), this means that each node $S_{i}$ can send data over a randomly oriented communication sector $\Phi_{i}$ of fixed angle $\alpha \in[0,2 \pi]$ degrees. The case with $\alpha=2 \pi$ represents omnidirectional communication for the RGG [5]. The receiving photo-detector is omni-directional, and thus receives data from any direction. This implies that node $S_{i}$ may directly talk to $S_{j}$ (denoted $S_{i} \rightarrow S_{j}$ ) if and only if $\operatorname{Pos}_{j} \in \Phi_{i}$. However, $S_{j}$ can only talk to $S_{i}$ via a multi-hop back-channel or reverse route, with other nodes in the network acting as routers along the path (unless of course $\operatorname{Pos}_{i} \in \Phi_{j}$ ). In the illustration of Figure 2(a) this reverse route is: $S_{j} \rightarrow S_{a} \rightarrow S_{b} \rightarrow S_{c} \rightarrow S_{i}$. Figure 2(b) simulates a sample flat OSN with $n=500$ nodes, $\alpha=2 \pi / 9$ radians, $r=\sqrt{\log (n) / n}$ as suggested in [1], and deployment region $[0,1]^{2}$. Note that communication sectors outside the deployment region are ignored, and do not contribute to network connectedness.

\section{B. Hierarchical Network Setup and Clustering}

Hierarchy in the OSN is achieved by employing a passive optical device known as the corner cube retro-reflector (CCR) [4]. A CCR is a simple optical device composed of three mutually perpendicular mirrors, which reflects incident light back to source. By mis-aligning one mirror of the CCR, a sensor node can transmit an on-off keyed signal to the base station [2]. When used to modulate an interrogating beam from the base station, the CCR yields huge energy savings compared to active laser communication [3]. CCR communication is passive and bi-directional between a node and the base station, and is especially attractive because all the optical energy for communication is supplied by the base station, with negligible energy used for the modulating circuitry of the CCR on the node. CCRs are great for OSNs due to their small size, ease of operation and negligible power consumption.

In addition to optical trans-receivers, all the OSN nodes are equipped with CCR's. After random deployment, a fraction of nodes $\{C H\} \in\left\{S_{n}\right\}$ called cluster heads, will have their CCR's oriented such that they have a communication (lineof-sight) path with the base station, and can thus employ their CCR's to exploit the advantages of passive bi-directional 

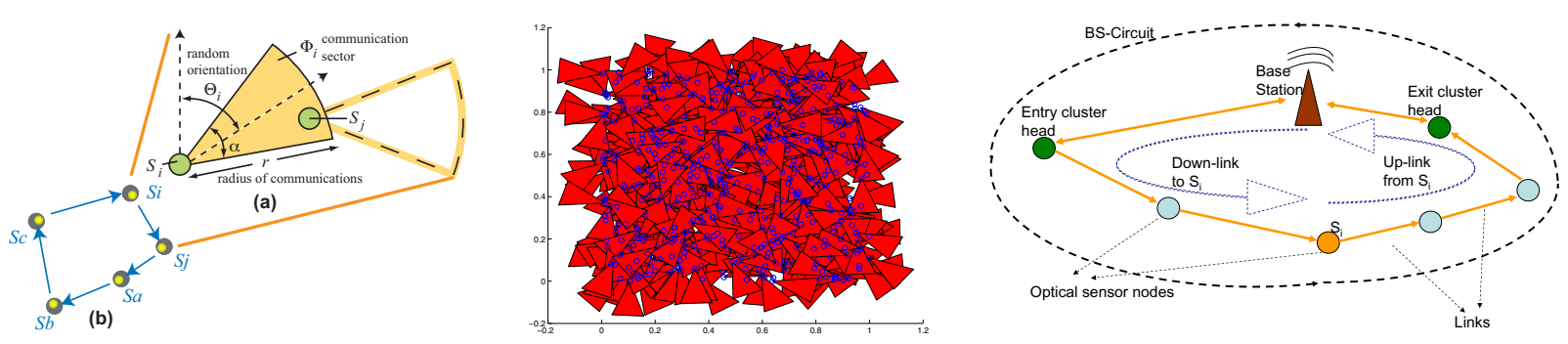

Fig. 2. (a) Node $S_{j}$ can only hear node $S_{i}$ if it falls into $S_{i}$ 's communication section. (b) However $S_{j}$ talks to $S_{i}$ via the back channel $S_{j} \rightarrow S_{a} \rightarrow S_{b} \rightarrow S_{c} \rightarrow S_{i}$. (c) An Optical Sensor Network graph showing connectivity and coverage concepts for $n=500$ nodes. (d)Illustrating the BS-circuit path with the down-link from the base station to node $S_{i}$ via the entry clusterhead, and the uplink path from $S_{i}$ to the base station via the exit clusterhead.

communication with the base station (see [2] for details). Let $P_{C H}$ be the probability that a node is a cluster head. There are $m \cong n P_{C H}$ cluster heads, and we designate cluster head nodes $S_{i}^{*}$ as $C H_{j}: j=1, \cdots m$. Without loss of generality, assume CCRs are oriented in the same direction as a nodes communication section. The random orientation of a node and the location of the base station determines the set of cluster heads $\{\mathrm{CH}\}$, which are uniformly distributed in the network.

Cluster heads $(\mathrm{CHs})$ forward/receive data to/from the base station without adversely depleting their energy resources. This leads to a natural hierarchy in which nodes route data to the upwards 'closest' $\mathrm{CH}$ for onward forwarding to the base station (uplink), or receive data or broadcasts from the base station (downlink) via another downwards closest $\mathrm{CH}$. All the possible uplink and downlink paths through any node constitute the node's BS-circuits. Figure 2(c) illustrates uplink and downlink paths from/to a $S_{i}$ through the exit/entry CHs.

\section{Graph Theoretic Setup}

We model the $n$-node OSN topology as a directed graph $G_{n}=\left(\mathcal{S}_{n}, \mathcal{E}, \Theta, r, \alpha\right)$ consisting of a vertex node set $\mathcal{S}_{n}$ and edge set $\mathcal{E}$, where every edge is an ordered pair of distinct nodes. $\mathcal{E}$ is represented as the $n \times n$ adjacency matrix of $G_{n}$ with one row and one column for every node, where:

$$
\begin{aligned}
\mathcal{E}(i, j)_{1 \leq i, j \leq n} & =1 \text { if } \operatorname{Pos}_{j} \in \Phi_{i} . \\
& =0 \text { otherwise. }
\end{aligned}
$$

indicates that there is, or there is not, an edge between $S_{i}$ and $S_{j} . \mathcal{E}(i, i)=0 \quad \forall i$ disallows self loops, and directionality implies $\mathcal{E}(i, j) \neq \mathcal{E}(j, i)$ necessarily, $\forall i, j$. $\Theta$ represents the orientation vector of $\mathcal{S}_{n}$, while $r$ and $\alpha$ (assumed to be the same for all nodes) are the respective radius and angle of $\Phi$.

Let us define node $S_{i}$ 's forward neighborhood $\left\{S_{i}^{+}\right\}$as the set of nodes that $S_{i}$ can talk to, i.e., $\left\{S_{i}^{+}\right\}=\left\{S_{k}\right\}, \forall k$ : $\mathcal{E}(i, k)=1$ or $S_{i} \rightarrow\left\{S_{k}\right\}$. Nodes in $\left.\left\{S_{i}^{+}\right\}\right)$are called $S_{i}$ 's successors, and the cardinality, $\# S_{i}^{+}$is equivalent to $S_{i}$ 's out degree. $S_{i}$ is forward-isolated if $\# S_{i}^{+}=0$. The expected out degree of $G_{n}$ denoted as $S^{+}$is $E\left(S_{i}^{+}\right) \simeq 1 / n \sum_{i=1}^{n} \# S_{i}^{+}$as $n \rightarrow \infty$, where $E($.$) is the expectation operator. Similarly,$ $S_{i}$ 's predecessors are nodes in its backward neighborhood, i.e., nodes who $S_{i}$ can hear, defined as $\left\{S_{i}^{-}\right\}=\left\{S_{h}\right\}, \forall h$ : $\mathcal{E}(h, i)=1$. The cardinality $\# S_{i}^{-}$is to $S_{i}^{\prime} s$ in degree, and $S_{i}$ is backward-isolated if $\# S_{i}^{-}=0$. The expected out degree of
$G_{n}$ is $S^{-}=E\left(S_{i}^{-}\right)$. The out and in degrees of $S_{i}$ is the sum along the $i^{\text {th }}$ row and $i^{\text {th }}$ column of $\mathcal{E}$ respectively.

\section{Connectivity Analysis}

\section{A. Forward Connectivity Analysis}

Consider the following $r$ and $\alpha$ assignment problem for the OSN: Given $G_{n}$, the objective is to find values of $r$ and $\alpha$, such that with at least probability $p_{f}, G_{n}$ is forward-connected. LEMMA 1: $G_{n}$ is forward-connected with at least probability $p_{f}$ if area of the communication sector $A(\Phi)$ is bounded as:

$$
\frac{\alpha r^{2}}{2} \geq \frac{-\ln \left(1-p_{f}^{1 / n}\right)}{n}
$$

PROOF: We employ quadrat statistical methods which is an approach taken to quantify spatial point patterns (see Chapter 8 of [13] $)^{1}$. Under this model, quadrats of random location and orientation are sampled, the number of events in the quadrat are counted, and statistics derived from the counts. It is well known [13] that the number of points located in a region $A$, follows a Poisson distribution of parameter $\lambda|A|$, where $|A|$ represents region $A$ 's area, and $\lambda$ is the intensity of the Poisson process. In the OSN with $\Phi$ as quadrats, the number of sensors located in $\Phi$ has a Poisson distribution with mean $\frac{\alpha n r^{2}}{2}$, so that the probability distribution function (pdf) of the number of nodes in each node's communication sector (i.e., $\# S^{+}$) is:

$$
{ }^{2} P\left(S^{+}=k\right)=\frac{e^{\frac{-\alpha n r^{2}}{2}}\left(\frac{\alpha n r^{2}}{2}\right)^{k}}{k !}
$$

and the probability that each node has at least one node in its communication sector (i.e. $P\left\{S_{i}^{+}\right\} \neq$null $\forall i$ ) is:

$$
P\left(S^{+} \geq 1\right)=\sum_{k=1}^{\infty} \frac{e^{\frac{-\alpha n r^{2}}{2}}\left(\frac{\alpha n r^{2}}{2}\right)^{k}}{k !}=1-e^{\frac{-\alpha n r^{2}}{2}}
$$

Assuming statistical independence of quadrats ${ }^{3}$, the probability that all $n$ nodes have at least one forward neighbor $p_{f}$ is:

$$
p_{f}=\left(\begin{array}{c}
n \\
n
\end{array}\right) P\left(S^{+} \geq 1\right)^{n} P\left(S^{+}=0\right)^{0}=\left(1-e^{\frac{-\alpha n r^{2}}{2}}\right)^{n}
$$

Lemma 1 follows by a change of subject for $A(\Phi)$ in Equation 4 , and generalizes the results of [7] for $\alpha=2 \pi$.

\footnotetext{
${ }^{1}$ Quadrats are bounded regions of any possible shape including $\Phi$.

${ }^{2}$ derived by employing series representation of the exponential function.

${ }^{3}$ Statistical independence of quadrants is reasonable since for numbers $a$ and $b$ the events $S_{i}^{+} \leq a$ and $S_{j}^{+} \leq b, \forall i, j$ are independent.
} 


\section{B. Joint Minimization of $r$ and $\alpha$}

Consider the following $r$ and $\alpha$ assignment problem: Given $G_{n}$, the objective is to find the jointly minimum sector range $r_{\min }$ and angle $\alpha_{\min }$, such that with probability at least $p_{f}, G_{n}$ is forward-connected. One motivation for jointly minimizing $\alpha$ and $r$ is to minimize the cost of the optical node such that $G_{n}$ is forward-connected with at least probability $p_{f}$. This is because energy dissipated at the optical transmitter is proportional to both the radius and angle of communication, since the received power at the optical receiver is [3]:

$$
P_{r e c^{\prime} v d}=P_{\text {trans }^{\prime} d} \times \frac{A_{\nu}^{2} \times 10^{-a . \nu / 10}}{\left[A_{t}^{2}+\alpha \nu\right]^{2}}
$$

where $P_{\text {trans }} d$ is the transmitter power, $a$ is atmospheric attenuation factor based on environmental conditions, $A_{r}$ and $A_{t}$ are the receiver and transmitter aperture diameter, $\alpha$ is beam divergence, and $\nu \leq r$ is the distance between optical transmitter and receiver. All the factors except $r$ and $\alpha$ are controlled by the system designer or obtained from site measurements. The only tunable parameters are $r$ and $\alpha$, both of whose squared values are approximately (inversely) proportional to $P_{\text {trans }}$ d .

Jointly minimizing $r$ and $\alpha$ results in the transmit energy being diffused in the smallest area, while maintaining connectivity. In addition, small beam angles are desirable as they yield higher security gains by reducing the risk of signal interception or denial of service attacks [15]. Previous work has considered optimizing $r$ for an $n$-node RGG, and in a directional network [1] using a pre-determined $\alpha$, usually chosen arbitrarily. In comparison, our approach yields an overall optimal system in terms of energy as seen from Equation 5.

LEMMA 2: Given that $A(\Phi)$ in $G_{n}$ is constrained as in Lemma 1, the jointly minimum values for $r$ and $\alpha$ of $\Phi$ are:

$r_{f \min }=\left[\frac{-4 \ln \left(1-p_{f}^{1 / n}\right)}{n}\right]^{\frac{1}{3}} \alpha_{f \min }=\left[\frac{-4 \ln \left(1-p_{f}^{1 / n}\right)}{8 n}\right]^{\frac{1}{3}}$

PROOF: Out of the set of values $(\alpha, r)$ that satisfy the constraint $A(\Phi)=\frac{-\ln \left(1-p_{f}^{1 / n}\right)}{n}$ for connectivity of the OSN, we seek the one which gives the least value for both $\alpha$ and $r$. This translates to a constrained minimization of the absolute value of the difference $|r-\alpha|$. Since $r$ and $\alpha$ are positive real numbers, we can minimize the sum of the two variables subject to the constraint. The minimax optimization is stated:

$$
\min _{(r, \alpha)} \max r+\alpha \quad \text { subject to } \quad \frac{\alpha r^{2}}{2}+\frac{\ln \left(1-p_{f}^{1 / n}\right)}{n}=0
$$

To solve this problem, we construct the Lagrange function $L(\alpha, r)$ employing the Lagrange multiplier $\beta$ as:

$$
L(\alpha, r)=r+\alpha+\beta\left(\alpha r^{2}+\frac{2 \ln \left(1-p_{f}^{1 / n}\right)}{n}\right)
$$

The simultaneous equations for solutions $\alpha_{\min }$ and $r_{\min }$ are:

$$
\frac{\partial L}{\partial \alpha}=0=1+\beta r^{2} \quad \text { and } \quad \frac{\partial L}{\partial r}=0=1+2 \alpha \beta r
$$

in which a simple change of variables yields $\alpha_{\min }=\frac{1}{2} \sqrt{-\frac{1}{\beta}}$ and $r_{\text {min }}=\sqrt{-\frac{1}{\beta}}$. We obtain the value of $\beta$ by substituting for $\alpha_{f \min }$ and $r_{f \min }$ in the constraint of Equation 7, to obtain:

$$
\beta=-\left[\frac{4 \ln \left(1-p_{f}^{1 / n}\right)}{n}\right]^{-2 / 3}
$$

Simple substitutions of Equation 10 into Equation 9 give the desired minimal values for $r_{f \min }$ and $\alpha_{f \min }$ in Lemma 2 .

\section{Backward Connectivity of the OSN}

The backward connectivity problem is analogous to an area coverage problem, in which a location is covered by a node if it lies within the nodes communication sector. Obviously, a covered node is backward-connected, (i.e., $\left\{S_{i}^{-}\right\} \neq 0, \forall i$ ).

LEMMA 3: $G_{n}$ is backward-connected with probability at least $p_{b}$ exactly equal to $p_{f}$.

PROOF: The proof of lemma 3 is constructed from concepts in stochastic geometry [14], similar to the proof of area coverage for RGG's in [8]. The probability that a point does not lie within any of the $n$ sensor node's communication sector $\Phi$ is $(1-A(\Phi))^{n}[14]$. For $[0,1]^{2}$ and $r<<1$, we have that $P$ (a point in $G_{n}$ is not covered) is:

$$
E(1-A(\Phi))^{n} \cong e^{-A(\Phi) \cdot \mathcal{E}(n)}=e^{\frac{-n \alpha r^{2}}{2}}
$$

Therefore, the probability that a node in the OSN is backwardconnected is: $p_{b}=1-e^{\frac{-n \alpha r^{2}}{2}}=p_{f}$ Even though analytic results give $p_{b}=p_{f}$, simulations show that number of backwardisolated nodes is always less than or equal to the number of forward-isolated nodes. We believe this is due to boundary effects, which has more impact on forward connectivity [15].

\section{D. fb-connectivity: Necessary but insufficient condition for} connectivity of the OSN

LEMMA 4: $G_{n}$ is fb-connected with at least probability $p_{f b}=p_{f} * p_{b}=\left(1-e^{\frac{-n \alpha r^{2}}{2}}\right)^{2 n}$, with corresponding jointly minimum values of $r$ and $\alpha$.

PROOF: Result follows from product of Lemmas 1 and 3.

\section{Hierarchical CONNECTIVITY: SUfFicient CONDITION FOR CONNECTIVITY OF THE CLUSTERED OSN}

Connectivity in ad hoc networks ensures the existence of a path between any two nodes in the network. However, the communication model for sensor networks is mainly base station-to-nodes (flooding) or nodes-to-base station (gathering) [15]. Therefore, connectivity in sensor networks should be hierarchical to reflect this property. In this section, we show that BS-connectivity is sufficient for OSN connectivity.

\section{A. BS-circuit connectivity}

$G_{n}$ is defined as BS-connected if every node is included in at least one BS-circuit. Practically, we interpret a BSconnected OSN as one in which with high probability, every node has at least one uplink path to a cluster head (uplink connectivity), and one down-link path from a cluster head 
(down-link connectivity). BS-connectivity analysis is useful in providing bounds on node connectivity, and in security analysis that evaluate the effect of a compromised node its descendants or members of its BS-circuit.

We define $G_{n}$ as $\delta$-uplink/downlink connected if for every node, there exists at least one descendant/ancestor within $\delta$ generations, which is a $C H$. That is, $S_{i} \notin\{C H\}$ is $\delta$-uplink connected if it has at least one uplink path to an exit cluster head within $\delta$ hops. $\delta$-downlink connected is similarly defined. The constant $\delta$ represents the network diameter, used to avoid excessively long paths. Obviously, if $S_{i} \in\{C H\}$, then it is connected and we are done. If none of $S_{i}$ 's descendants (within $\delta$ generations), is a $C H, S_{i}$ cannot possibly find any uplink path to the base station and is disconnected from the network. Given $\delta$ and $P_{C H}$, our goal is to determine $p_{u}$, the probability that $G_{n}$ is $\delta$-uplink/downlink connected. We provide analysis for the uplink connectivity and then generalize for the downlink.

We employ the Galton-Watson Branching Process [16] (GWBP) to model the uplink tree rooted at any arbitrary node. GWBP were introduced by Francis Galton in 1889 as a mathematical model for the propagation of family names. Let $S_{i}$ independently give rise to the set of forward neighbors $\left\{S_{i}^{+}\right\}$with cardinality $\xi_{1} \equiv \# S_{i}^{+}$. The $\xi_{1}$ nodes are $S_{i}$ 's first generation (level $L_{1}$ ) offspring. Let the $\xi_{1}$ successors of $S_{i}$ independently give rise to $\xi_{2}$ second generation level $L_{2}$ offspring of $S_{i}$, denoted $\left\{S_{i}^{+}\right\}^{2}$, and so on. We denote $\xi_{k}$ as the number of $S_{i}$ 's $k^{t h}$ generation offspring with the set of nodes $\left\{S_{i}^{+}\right\}^{k}$, where $k=1,2, \cdots \delta$. Let $D_{i}=\sum \xi_{k}$ be the total number of $S_{i}$ 's descendants.

The random variables $\xi_{i}$ for $i=1 \cdots \delta$ representing the offspring distribution $P\left(\xi_{i}=K\right)$ of nodes for generation $L_{i}$, form a discrete-time Markov chain (since $P\left(S_{i}^{+k} \mid S_{i}^{+(k-1)} \cdots S_{i}^{+1}\right)=P\left(S_{i}^{+k} \mid S_{i}^{+(k-1)}\right)$, which takes values in the set of nonnegative integers with transition probabilities $P\left(\xi_{i+1}=k \mid \xi_{i}=m\right)=P\left(\xi_{1}=k\right)^{* m}$. Here $P\left(\xi_{1}=\right.$ $k)^{* m}$ denotes the $m^{t h}$ convolution power of $P\left(\xi_{1}=K\right)$. Therefore, conditional distribution of $\xi_{i+1}$ given $\xi_{i}=m$ is the distribution of a sum of $m$ i.i.d. random variables each with distribution $P\left(\xi_{i}\right)$. In our case, we can build the GWBP tree using $\xi_{1}$, since we know $P\left(\xi_{1}\right) \sim \operatorname{Poisson}\left(\frac{n \alpha r^{2}}{2}\right)$.

Now, considering the occurrence of a successor in each generation as independent events (since nodes may repeat), the probability that at least one of $S_{i}$ 's level $L_{1}$ offspring is a $C H$ is: $\sum_{k=0}^{\infty} P\left(\xi_{1}=k\right) \cdot\left[1-P\left(\left\{S_{i}^{+}\right\} \notin\{\mathcal{C H}\} \mid \xi_{1}\right)\right]$

$$
=\sum_{k=0}^{\infty}\left[\frac{e^{-\lambda}(\lambda)^{k}}{k !} 1-\left(1-P_{C H}\right)^{k}\right]
$$

Similarly, for the second generation, the probability that at least one of $S_{i}$ 's level $L_{2}$ offspring is a $C H$ is given as: $\sum_{m=0}^{\infty} \sum_{k=0}^{\infty} P\left(\xi_{1}=k\right) \cdot P\left(\xi_{2}=m \mid \xi_{1}=k\right) \times$
$\left[1-P\left(\bigcup_{i=1}^{2}\left\{S_{i}^{+}\right\}^{i} \notin\{\mathcal{C H}\} \mid \xi_{1}=k, \xi_{2}=m\right)\right.$

$$
=\sum_{m=0}^{\infty} \sum_{k=0}^{\infty} \frac{e^{-\lambda(m+1)}\left(m \lambda^{2}\right)^{k}}{k !^{2}}\left[1-\left(1-P_{C H}\right)^{k+m}\right]
$$

Therefore, for path lengths $\leq \delta$, the probability that $G_{n}$ is $\delta$-uplink connected $p_{u}=\left[\bar{P}\left(\exists\right.\right.$ at least one $\bigcup_{i=1}^{\delta}\left\{S_{i}^{+}\right\}^{i} \in$
$\{\mathcal{C H}\})]$ for all $n\left(1-n P_{C H}\right)$ non cluster head nodes is:

$$
\begin{aligned}
\sum_{k_{\delta}=0}^{\infty} \cdots \sum_{k_{2}=0}^{\infty} \sum_{k_{1}=0}^{\infty} \frac{e^{-\lambda\left(\sum_{p=1}^{\delta-1} k_{p}+1\right)}\left(\prod_{p=1}^{\delta-1} k_{p}^{k_{p-1}} \lambda^{2}\right)}{k_{1}^{2} ! \prod_{p=2}^{\delta-1} k_{p} !} \\
\times\left[1-\left(1-P_{C H}\right)^{\sum_{p=1}^{\delta} k_{p}}\right]^{n\left(1-P_{C H}\right)}
\end{aligned}
$$

Note that $P_{C H} \rightarrow 1$ leads to the trivial sufficient condition that $G_{n}$ is uplink-connected. Similar analysis for downlink connectivity with $p_{b}=p_{f}$,yields that the probability that $G_{n}$ is $\delta$-downlink connected $p_{d}$ equals $p_{u}$. A necessary and sufficient condition for $G_{n}$ to be hierarchical connected is that the network is both downlink and uplink-connected with probability $p \leq p_{d} \times p_{u}$, easily derived from Equation 14 .

\section{CONCLUSIONS}

This paper provides analytical insights for probabilistic bounds on network parameters for hierarchical connectivity in OSNs. Our novel approach considers directionality. We have derived expressions for the probability of the hierarchical connectivity of the OSN and jointly minimized $r$ and $\alpha$ values.

\section{REFERENCES}

[1] J. Diaz, J. Petit, and M. Serna, A random graph model for optical networks of sensors, In IEEE Transactions on Mobile Computing, vol. 2, no. 3, pp. 186196, July- September 2003.

[2] J. M. Kahn, R. H. Katz, and K. S. J. Pister. Next century challenges: Mobile networking for smart dust. InProc. ACM/IEEE Int. Conf. on Mobile Computing and Networking, Washington, pp. 271278, Aug 1999.

[3] J. Hill, R. Szewczyk, A. Woo, S. Holler, D. Culler and K. Pister. System architecture directions for networked sensors. Proc. of the ninth Int conf. on Architectural support for programming languages and operating systems, pages 93-104, 2000.

[4] S. Teramoto and T. Ohtsuki. Optical wireless sensor network system using corner cube retroreflectors. 2005. EURASIP Journal of Applied Signal Processing 1, 39-44. (Mar. 2005).

[5] E. Kranakis, D. Krizanc and E. Williams, Directional versus Omnidirectional Antennas for Energy Consumption and k-Connectivity of Networks of Sensors. In Proceedings of OPODIS 2004, pp. 357-368, Teruo Higashino (ed.), SVLNCS, Vol. 3544.

[6] P. Gupta and P. R. Kumar Critical power for asymptotic connectivity, In Proc. of IEEE CDC, Tampa, USA, 1998.

[7] C. Bettstetter, On the minimum node degree and connectivity of a wireless multihop network, In Proc. of the 3rd ACM Int. symposium on Mobile ad hoc networking \& computing pp. 80-91, Switzerland, 02.

[8] B. Liu and D. Towsley, A Study of the Coverage of Large-scale Sensor Networks, In Proc. The 1st IEEE International Conference on Mobile Ad-hoc and Sensor Systems October 24-27, 2004, Florida, USA

[9] M. D. Penrose On k-Connectivity for a geometric random graph In Random Structures and Algorithms. 15(2): pp. 145-164, 1999.

[10] F. Xue and P. R. Kumar, The number of neighbors needed for connectivity of wireless networks, Wireless Networks, vol. 10, no. 2, pp. 169-181, 2004.

[11] S. Song and D. L. Goeckel and D. Towsley, An Improved Lower Bound to the Number of Neighbors Required for the Asymptotic Connectivity of Ad Hoc Networks, IEEE Transactions on Information Theory, 2005.

[12] H. Ma and Y. Liu, On Coverage Problems of Directional Sensor Networks, in Proceedings of the International Conference on Mobile Ad-hoc and Sensor Networks (MSN), Wuhan, China, Dec. 2005.

[13] N. Cressie, Statistics for Spatial Data, John Wiley \& Sons, 1991.

[14] P. Hall, Introduction to the Theory of Coverage Processes. John Wiley \& Sons, 1988.

[15] U. Ndili Okorafor and D. Kundur. OPSENET: A Security Enabled Routing Scheme for a System of Optical Sensor Networks, Proc. International Conf on Broadband Comm, Networks, and Systems (BROADNETS), San Jose, California, Oct 2006.

[16] P. Guttorp, Statistical Inference for Branching Processes. John Wiley \& Sons, Inc., New York 1991. 